\title{
Predição da codeleção 1p/19q utilizando atributos radiômicos e aprendizado de máquina
}

\author{
Tony Alexandre Medeiros da Silva, Guilherme de Souza e Cassia, Francisco Assis de Oliveira Nascimento e \\ João Luiz Azevedo de Carvalho
}

\begin{abstract}
Resumo - Neste trabalho, demonstramos a predição da codeleção dos cromossomos $1 p / 19 q$ em gliomas de baixo grau, pois representa um fator prognóstico positivo ao tratamento quimioterápico. A partir da análise de imagens de ressonância magnética, usamos aprendizado de máquina baseado em atributos radiômicos extraídos das imagens utilizando a biblioteca PyRadiomics. A classificação foi realizada usando dois modelos de algoritmos de classificação: a regressão logística (RL) e a floresta aleatória (RF). Utilizamos a análise de componentes principais para redução de dimensionalidade dos atributos. Após a execução dos algoritmos RL e RF no conjunto de treinamento, os modelos gerados foram validados no conjunto de validação, obtendo $83,24 \%$ e $84,67 \%$ de acurácia, respectivamente. Esses resultados se mostraram bastante promissores na predição do status de codeleção dos cromossomos $1 \mathrm{p} / 19 \mathrm{q}$.
\end{abstract}

Palavras-Chave - radiômica, gliomas de baixo grau, codeleção $1 p / 19 q$, regressão logística e floresta aleatória.

Abstract - In this work, we demonstrate the prediction of codeletion of chromosome $1 \mathrm{p} / \mathbf{1 9 q}$ in low-grade gliomas, as it represents a positive prognostic factor for chemotherapy treatment. From the analysis of magnetic resonance images, we use machine learning based on radiomics features extracted from the images using the PyRadiomics library. The classification was performed using two models of classification algorithms: logistic regression ( $R L)$ and random forest $(R F)$. We use the principal component analysis to reduce the dimensionality of the features. After the execution of the RL and RF algorithms in the training set, the generated models were validated in the validation set, obtaining $83.24 \%$ and $84.67 \%$ accuracy, respectively. These results showed to be very promising in the prediction of the codeletion status of chromosome 1p/19q.

Keywords - radiomics, low-grade gliomas, co-deletion 1p/19q, regression logistic and random forest.

\section{INTRODUÇÃO}

A ressonância magnética $(\mathrm{RM})$ é o principal método diagnóstico por imagem para tumores cerebrais [1]. Nesse sentido, a radiômica se propõe a ser utilizada como ferramenta de extração de atributos quantitativos da RM permitindo a mineração de informações não visuais, que podem representar características histopatológicas e genéticas do tumor, possibilitando o diagnóstico molecular, a avaliação quanto ao grau tumoral e prognóstico, além de auxiliar na tomada de decisão clínica, guiando tratamentos e o mapeamento précirúrgico [2].

Os gliomas de baixo grau (LGG), são um grupo de tumores heterogêneos e com características histológicas potencialmente agressivas. De acordo com sua atualização de 2016, a OMS faz

Tony Alexandre Medeiros da Silva, Departamento de Engenharia Elétrica, Universidade de Brasília, Brasília-DF, e-mail: tonyufu2005@gmail.com; Guilherme de Souza e Cassia, Departamento de Diagnóstico por Imagem, DF Star Hospital/Rede D’Or, Brasília-DF, e-mail: guilhermecassia@gmail.com; Francisco Assis de Oliveira Nascimento, Departamento de Engenharia Elétrica, Universidade de Brasília, Brasília-DF, e-mail: assis@ene.unb.br; João Luiz Azevedo de Carvalho, Departamento de Engenharia Elétrica, Universidade de Brasília, Brasília-DF, e-mail: joaoluiz@pgea.unb.br. recomendações para que se utilize uma classificação integrativa dos tumores, considerando características histopatológicas e moleculares, permitindo assim um melhor manejo clínico. Sendo assim, a OMS destaca a importância de biomarcadores genéticos em tumores cerebrais como, a codeleção cromossômica 1p/19q na classificação dos LGGs [3].

A avaliação da codeleção cromossômica $1 p / 19 q$ requer a análise histopatológica de um fragmento do tumor, retirado de maneira invasiva, por biópsia ou cirurgia, com eventual morbiletalidade. Portanto, estudos convencionais apresentam crescente interesse no desenvolvimento de técnicas não invasivas para classificação de alterações tumorais. Na literatura já foram desenvolvidos alguns estudos com o objetivo de prever o status da codeleção 1p/19q em LGGs utilizando aprendizado de máquina em seu fluxo de trabalho [4,5,6]. É importante destacar que essa validação se faz necessária em um conjunto de dados desconhecido ao algoritmo, possibilitando-o encontrar uma melhor solução e possivelmente fornecer a perspectiva de uma classificação ou predição aceitável para adoção clínica no futuro. Neste estudo, avaliamos a possibilidade de uma classificação não invasiva de gliomas de baixo grau, com respeito ao status da codeleção cromossômica $1 \mathrm{p} / 19 \mathrm{q}$, utilizando atributos radiômicos e aprendizado de máquina.

\section{MATERIAIS E MÉTODOS}

\section{A. Características do dataset}

Utilizamos o dataset LGG-1p19qDeletion disponível no The Cancer Imaging Archive (TCIA) [7]. Ele contém imagens préoperatória de 150 pacientes. São 96 pacientes com status de codeletados e 54 pacientes com status de não-codeletados. Neste estudo, não foi necessário obter aprovação de comité ético, por se tratar de um conjunto de imagens livremente disponível para fins científicos Achados clínicos, conforme exemplifica a Figura 1, sugerem um diagnóstico genético; porém, todos os pacientes foram submetidos a avaliação através de estudo histopatológico por meio de biopsia para confirmar o status de codeleção 1p/19q. Dos LGGs que fazem parte do estudo temos: 17 astrocitomas, 45 oligodendrogliomas e 88 oligoastrocitomas. A Figura 2 apresenta a divisão do status de codeleção versus LGGs desse dataset. Akkus et al. [4] afirmam que as imagens foram adquiridas seguindo um protocolo consistente, com ponderação T2 e cortes de $1 \mathrm{~mm}$ e $3 \mathrm{~mm}$ em aparelhos de $1,5 \mathrm{~T}$ e $3 \mathrm{~T}$. A delimitação das regiões de interesse também seguiu um protocolo de segmentação, através de uma ferramenta semiautomática desenvolvida pelos autores.

\section{B. Pré-processamento}

Extração dos atributos: Após o download do dataset, foi necessário realizar a conversão dos arquivos, que encontravamse no formato .nii, para o formato .nrrd. Para tal conversão utilizamos a ferramenta 3DSlicer [8]. Em seguida, utilizamos uma biblioteca de código aberto baseada em linguagem de 
programação Python para extração dos atributos quantitativos chamada PyRadiomics [9]. Ao ser acionada, a plataforma PyRadiomics executa quatro etapas principais: i) leitura das imagens e regiões segmentadas; ii) aplicação de filtros; iii) cálculo dos atributos dentro das classes selecionadas; e iv) retorno dos resultados.

Nesse estudo foram extraídos 100 atributos rotulados pela plataforma como originais (primeira ordem, forma e textura) conforme apresenta a Tabela 1.

Redimensionamento dos atributos: Na busca de técnicas para seleção de atributos, utilizamos a análise de componentes principais (PCA), a qual visa realizar uma redução de dimensionalidade linear em um espaço de alta dimensão e em seguida projetar os dados em um subespaço de menor dimensão [10]. Na prática, a PCA preserva as características essenciais dos dados (componentes com maior variância, após uma mudança de base), e remove as características menos essenciais (componentes com menor variância). Em estudos anteriores, essa técnica se apresentou relevante em resultados obtidos na etapa de validação dos dados [11]. Neste estudo foi realizado uma redução de dimensionalidade das 100 colunas de atributos para 8 colunas de atributos. As 8 novas componentes preservaram 94\% da energia dos dados, sendo os respectivos percentuais de energia: componente $1=47 \%$, componente $2=19 \%$, componente $3=13 \%$, componente $4=5 \%$, componente $5=3 \%$, componente $6=3 \%$, componente $7=2 \%$ e componente $8=2 \%$. (a)

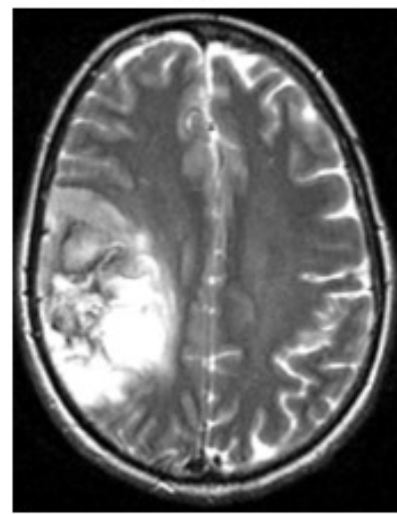

(b)

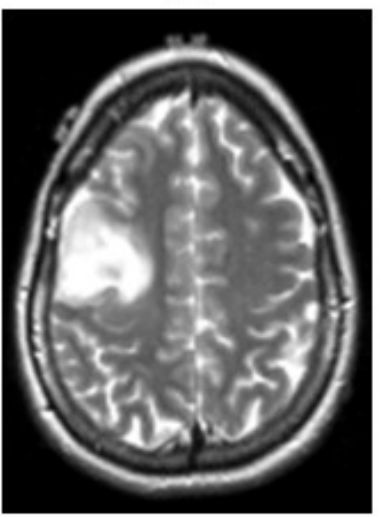

Fig. 1 Exemplos de LGGs: (a) grande lesão infiltrativa heterogênea frontoparietal direita, com áreas de baixa intensidade de sinal, sugerindo hemorragia ou calcificação, com diagnóstico de oligodendroglioma (codeleção $1 p / 19 q)$; e (b) lesão infiltrativa homogênea frontal direita com alta intensidade de sinal e diagnóstico de astrocitoma (ausência de codeleção 1p/19q).

Geração de novas instâncias: Como o dataset apresenta desbalanceamento no número de classes, essa ação foi de fundamental importância para melhor aprendizado do modelo na etapa de treinamento. Portanto, foi implementado uma técnica de aumento do número de instâncias (casos) através da adição de um ruído gaussiano com média igual a 0 , desvio padrão igual a 1 e potência igual a 0.2 , sendo que a potência do ruído deve ser baixa para que os dados não se encontrem fora da faixa de grandeza dos demais atributos. Técnicas como transformações geométricas (flipping, rotação, translação, corte, zoom, dentre outras) e transformações fotométricas, que atuam na mudança do valor do pixel, como por exemplo adição de ruído, filtragem com kernel e random erasing, são comumente utilizadas [12].
Os autores de [13] utilizaram adição de ruído em 9 bases de dados e comprovaram que essa técnica deixa o aprendizado de máquina mais eficaz no aprendizado de características robustas. Esta etapa foi realizada usando a ferramenta FAON - Machine Learning Platform, que se encontra em desenvolvimento por um dos autores deste trabalho.

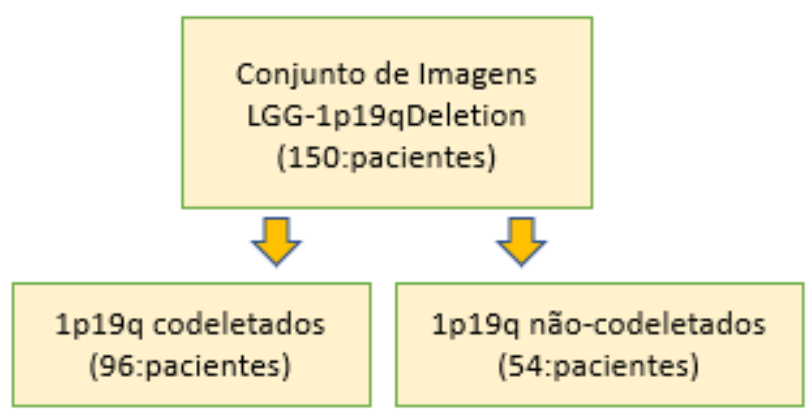

Fig. 2 Fluxograma da divisão do dataset LGG-1p19qDeletion.

Tab. 1 Quantidade de atributos radiômicos extraídos por classe.

\begin{tabular}{|l|c|}
\hline \multicolumn{1}{|c|}{ Classe de atributos [9] } & $\begin{array}{c}\text { quantidade } \\
\text { de atributos }\end{array}$ \\
\hline estatísticas de primeira ordem & 18 \\
\hline baseado em forma & 14 \\
\hline matriz de coocorrência de nível de cinza & 22 \\
\hline matriz de duração de nível de cinza & 16 \\
\hline matriz de tamanho de zona de nível de cinza & 16 \\
\hline matriz de dependência de nível de cinza & 14 \\
\hline
\end{tabular}

\section{Classificação}

O aprendizado de máquina tem evoluído e alcançado resultados significantes com relação ao reconhecimento de padrões. Neste estudo, para validação dos dados e predição do status da codeleção $1 \mathrm{p} / 19 \mathrm{q}$, foram selecionados 2 modelos [14]: a regressão logística (RL) e a floresta aleatória (random forest, ou RF), ambos classificadores com abordagem supervisionada.

A RL, por ser uma técnica estatística, produzirá, a partir de um conjunto de atributos previsores, um modelo que possa ser capaz de predizer uma saída categórica. Essa técnica mede a relação entre a variável dependente e variáveis independentes, estimando probabilidades, usando sua função logística subjacente.

$$
p=\frac{1}{1+e^{-y}}
$$

O método RF é baseado no conceito de ensemble learning, que é um processo de combinação de vários classificadores para resolver um problema complexo e melhorar o desempenho do modelo. Ele constrói um conjunto de árvores de decisão em vários subconjuntos a partir dos dados fornecidos e, ao invés de depender de uma única árvore de decisão, mescla os resultados dessas árvores com base na maioria dos votos para predizer um resultado de saída. A Figura 3 exemplifica seu funcionamento. 


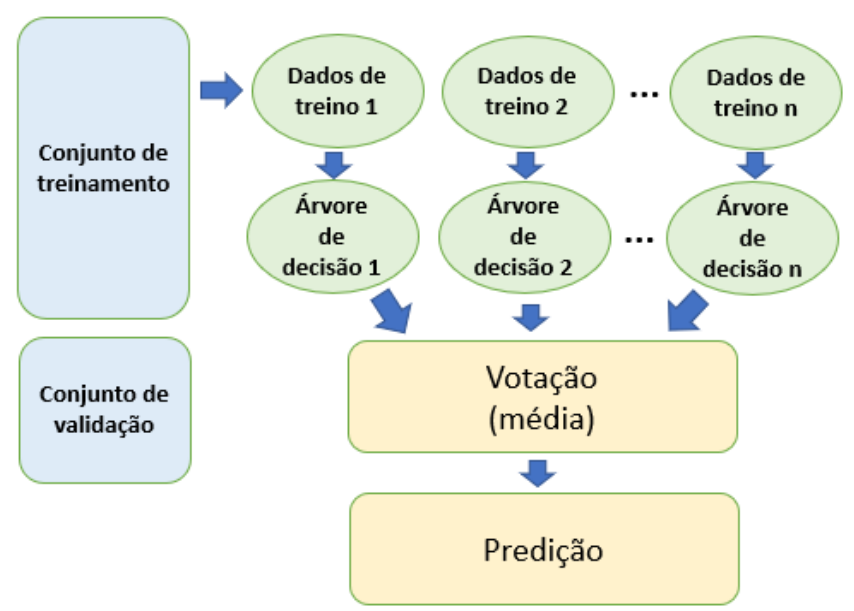

Fig. 3 Funcionamento do algoritmo RF.

Os algoritmos RL e RF também foram executados usando a plataforma FAON - Machine Learning Platform. Essa plataforma aproveita muito bem as unidades de processamento e acelera os algoritmos de aprendizado de máquina. Além disso, implementa vários outros algoritmos de classificação, regressão e clusterização.

Conforme distribuição do dataset (Figura 2), e descrição realizada pela etapa de redução de dimensionalidade (aplicação da PCA), o conjunto de dados composto por 150 casos e 8 atributos, foram divididos em 2 subconjuntos: $80 \%$ dos dados (equivalente a 120 casos) para treinamento e $20 \%$ dos dados (equivalente a 30 casos) para validação. Ao conjunto de treinamento foi aplicado a ação de geração de novas instâncias em mais 3 vezes, totalizando 480 casos para treinamento. No conjunto de dados de treinamento foi aplicado uma abordagem de validação cruzada para geração do modelo treinado. Essa técnica de validação cruzada (cross-validation, do inglês) é amplamente utilizada em trabalhos nos quais o objetivo é a predição de dados. Há na literatura uma discussão sobre o melhor tamanho de divisão dos conjuntos de dados, todavia, os tamanhos 5 e 10 tem se tornado o padrão ouro em termos práticos [15]. Nesse trabalho utilizamos uma validação cruzada de tamanho igual a 5. Ou seja, a plataforma divide os dados separados para treinamento em 5 grupos realizando o treinamento em 4 grupos e teste em 1 grupo. Essa ação é repetida pelo número de divisões na validação cruzada. A cada etapa uma pontuação é adquirida, e ao final das 5 etapas, uma média das pontuações e apresentada para avaliação da construção do modelo. A Figura 4 demonstra a utilização da validação cruzada em nosso trabalho. O modelo gerado é então utilizado para predizer a codeleção cromossômica $1 \mathrm{p} / 19 \mathrm{q}$ no conjunto de dados separado para validação.

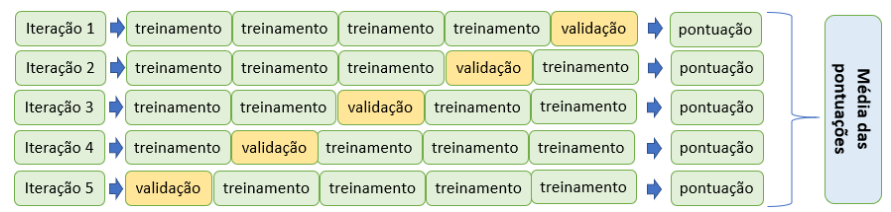

Fig. 4 Etapas de execução da validação cruzada.

Como se trata de um aprendizado supervisionado, ao executar o treinamento dos dados pelos algoritmos RL e RF, os mesmos vão relacionar os atributos previsores (entrada) e os atributos categóricos (saída), conforme a divisão do dataset em treinamento e validação.

\section{RESULTADOS}

A Tabela 2 apresenta o desempenho obtido pelos algoritmos RL e RF na classificação do status da codeleção cromossômica $1 \mathrm{p} / 19 \mathrm{q}$ no conjunto de validação. De acordo com o préprocessamento aplicado e parâmetros utilizados na etapa de classificação, ambos descritos anteriormente, aplicamos as seguintes métricas [15]: acurácia $(A c c)$, especificidade (Esp), sensibilidade (Sen) e precisão (Pre), calculadas como

$$
\begin{gathered}
A c c=\frac{v p+v n}{v p+f p+v n+f n} \\
E s p=\frac{v n}{v n+f p} \\
\text { Sen }=\frac{v p}{v p+f n} \\
\text { Pre }=\frac{v p}{v p+f p}
\end{gathered}
$$

em que $v p$ são os verdadeiros positivos, $v n$ são os verdadeiros negativos, $f p$ são os falsos positivos e $f n$ são os falsos negativos.

Tab. 2 Desempenho dos algoritmos RL e RF no conjunto de validação.

\begin{tabular}{|c|c|c|c|c|}
\hline Algoritmo & Acc (\%) & Esp (\%) & Sen (\%) & Pre (\%) \\
\hline RL & 83,24 & 87,52 & 81,43 & 94,91 \\
\hline RF & 84,67 & 88,28 & 83,45 & 95,94 \\
\hline
\end{tabular}

Os dois algoritmos utilizados para a classificação da codeleção cromossômica $1 \mathrm{p} / 19 \mathrm{q}$ apresentaram bons resultados. Porém, o algoritmo RF apresentou melhor resultado que o RL em todas as métricas avaliadas.

\section{DISCUSSÃO}

A biblioteca PyRadiomics se mostrou adequada para extração dos atributos radiômicos, tendo como fator negativo a necessidade de conversão do formato dos arquivos de DICOM (.dcm) ou NIFTI (.nii) para NRRD (.nrrd).

Um grande desafio para execução de algoritmos de aprendizado de máquina é a obtenção de um dataset com número suficiente de casos (instâncias). Os autores de [16] indicam uma relação de 10 a 15 instâncias para cada atributo radiômico utilizado. Devido a proposta de trabalhar com 100 atributos radiômicos em uma base com 150 casos, a utilização da PCA para redução da dimensionalidade do número de atributos se mostrou essencial para obtenção dos bons resultados obtidos.

Os modelos RL e RF foram capazes reconhecer os atributos radiômicos extraídos das imagens de $\mathrm{RM}$, com bom resultado. Isto foi alcançado usando uma técnica baseada em adição do ruído gaussiano para aumento do conjunto de dados para treinamento. As métricas de desempenho utilizadas - acurácia, sensibilidade, especificidade e precisão - foram escolhidas por serem amplamente utilizadas em publicações de trabalhos semelhantes, permitindo, assim, uma comparação mais adequada. 
Tab. 3 Resultados apresentados por trabalhos correlatos com o mesmo dataset (LGG-1p19qDeletion).

\begin{tabular}{|l|l|l|l|l|l|}
\hline Referência & Algoritmo & Acc (\%) & Esp (\%) & Sen (\%) & Pre (\%) \\
\hline $\begin{array}{l}\text { Akkus et } \\
\text { al [4] }\end{array}$ & SVM & 81,0 & 82,0 & 80,0 & \\
\hline $\begin{array}{l}\text { Lu et al } \\
{[17]}\end{array}$ & SVM & 80,0 & 66,7 & 100,0 & \\
\hline $\begin{array}{l}\text { Han et al } \\
{[18]}\end{array}$ & RF & 70,0 & 71,2 & 68,3 & \\
\hline $\begin{array}{l}\text { Kong et al } \\
{[19]}\end{array}$ & RF & 83,3 & 75,0 & 100,0 & \\
\hline $\begin{array}{l}\text { Van der } \\
\text { Voort et al } \\
{[20]}\end{array}$ & SVM & 69,3 & 61,7 & 73,2 & 78,7 \\
\hline $\begin{array}{l}\text { Shboul et } \\
\text { al [21] }\end{array}$ & XGBoost & & 85,0 & 75,0 & \\
\hline
\end{tabular}

* Algumas referências não apresentaram as métricas acurácia e precisão.

A Tabela 3 apresenta os resultados de outros trabalhos correlatos com utilização do dataset LGG-1p19qDeletion. Podese notar que os resultados obtidos em nosso trabalho superam os resultados da literatura. Em relação à precisão, métrica na qual pode-se encontrar a razão entre a quantidade de casos classificados corretamente como positivos e o total dos casos identificados como positivos, os algoritmos RL e RF demonstraram resultados bem superiores ao apresentado na referência [20]. Em relação a acurácia, métrica que nos diz de fato quanto dos casos foram classificados corretamente, independente da classe, o algoritmo RL apresenta pequena diferença a menor do que o valor obtido na referência [19]. Porém, o algoritmo RF obteve um resultado superior a todos os demais valores obtidos pelas referências apresentadas na Tabela 3. A sensibilidade, que representa no contexto do diagnóstico médico o percentual de acerto dos casos verdadeiros positivos, nosso resultado foi inferior apenas aos apresentados nas referências [17] e [19]; porém, em comparação com as demais referências apresentadas na Tabela 3, os valores obtidos em nosso trabalho apresentam de 5 a 8 pontos percentuais de melhora. Com relação à especificidade, que apresenta o percentual de acerto de casos verdadeiros negativos, ambos os algoritmos RL e RF apresentaram resultados superiores a todos os demais resultados apresentados nas referências listadas na Tabela 3 .

A Tabela 4 apresenta trabalhos correlatos que também realizaram a predição da codeleção cromossômica $1 \mathrm{p} / 19 \mathrm{q}$, porém em datasets diferentes do LGG-1p19qDeletion (utilizado em nosso trabalho e nos trabalhos listados na Tabela 3). Em comparação aos valores apresentados na Tabela 4, os algoritmos RL e RF se mostraram relevantes na identificação do status da codeleção cromossômica $1 \mathrm{p} / 19 \mathrm{q}$. Embora não tenham sido testados os mesmos conjuntos de dados aos respectivos datasets dos trabalhos referenciados na Tabela 4, mas o grupo de atributos radiômicos foram os mesmos. Nesse sentido, torna-se interessante uma comparação entre trabalhos que possuem o mesmo objetivo. Os métodos propostos apresentaram resultados superiores aos apresentados na literatura listada na Tabela 4, exceto pela métrica de sensibilidade obtida por Han et al [18] e por Kokal et al. [24]. Há de se destacar que os valores obtidos na acurácia e especificidade com a execução do algoritmo RL por Han et al [18] foram bem inferiores aos valores obtidos em nosso trabalho e aos demais trabalhos referenciados. Entende-se que o conjunto de dados utilizados em Han et al [18] não foi suficiente para que os atributos radiômicos extraídos pudessem predizer a codeleção cromossômica 1p/19q.

Tab. 4 Resultados apresentados por trabalhos correlatos em datasets diferentes.

\begin{tabular}{|l|l|l|l|l|l|}
\hline Referência & Algoritmo & Acc (\%) & Esp (\%) & Sen (\%) & Pre (\%) \\
\hline $\begin{array}{l}\text { Han et al } \\
{[18]}\end{array}$ & RL & 59,4 & 32,7 & 92,7 & \\
\hline $\begin{array}{l}\text { Decuyper } \\
{[22]}\end{array}$ & CNN & 83,3 & 82,0 & 80,0 & \\
\hline $\begin{array}{l}\text { Fukuma et } \\
\text { al. [23] }\end{array}$ & CNN & 79,8 & & & \\
\hline $\begin{array}{l}\text { Kokal et } \\
\text { al. [24] }\end{array}$ & MLP & & 75,8 & 87,5 & \\
\hline
\end{tabular}

* Algumas referências não apresentaram as métricas precisão, acurácia, especificidade e sensibilidade.

\section{CONCLUSÃO}

Este trabalho apresentou uma técnica não invasiva baseada em análise de atributos radiômicos extraídos de imagens de RM com ponderação em T2, PCA para redução de dimensionalidade, adição de ruído gaussiano para aumento do conjunto de dados de treinamento, e abordagens RL e RF para classificação -, que se mostraram eficazes para predizer a codeleção cromossômica 1p/19q em LGGs. A identificação da codeleção cromossômica $1 \mathrm{p} / 19 \mathrm{q}$ em pacientes com LGG representa uma oportunidade positiva de se determinar melhores decisões clínicas, cirúrgicas e terapêuticas. Essas tomadas de decisões são importantes, pois tumores codeletados (oligodendrogliomas) apresentam melhor resposta quimioterápica e, proporcionando aos respectivos pacientes uma maior sobrevida global, em comparação aos pacientes com tumores não-codeletados (astrocitomas).

\section{REFERÊNCIAS}

[1] Cha S. "Update on brain tumor imaging: from anatomy to physiology". AJNR Am J Neuroradiol. (2006);27(3):475-87

[2] Lambin P, Leijenaar R T H, Deist T M, Peerlings J, de Jong E E C, van Timmeren J, et al. Radiomics: the bridge between medical imaging and personalized medicine. Nat Rev Clin Oncol. (2017) 14:749-62. doi: 10.1038/nrclinonc. 2017.141

[3] Louis D N, et al. The 2016 World Health Organization Classification of Tumors of the Central Nervous System: a summary. Acta Neuropathol (2016) 131:803-820. DOI 10.1007/s00401-016-1545-1

[4] Akkus Z, Ali I, Sedlář J, Agrawal J P, Parney I F, Giannini C, Erickson B J. Predicting Deletion of Chromosomal Arms $1 p / 19 q$ in Low-Grade Gliomas from MR Images Using Machine Intelligence. J Digit Imaging. (2017) Aug; 30(4): 469-476. DOI: 10.1007/s10278-017-9984-3. PMCID: PMC5537096

[5] Han, Y., Xie, Z., Zang, Y. et al. Non-invasive genotype prediction of chromosome $1 \mathrm{p} / 19 \mathrm{q}$ co-deletion by development and validation of an MRI-based radiomics signature in lower-grade gliomas. J Neurooncol 140, 297-306 (2018). https://doi.org/10.1007/s11060-0182953-y

[6] Van der Voort, S. R. et al. Predicting the $1 \mathrm{p} / 19 \mathrm{q}$ co-deletion status of presumed low grade glioma with an externally validated machine learning algorithm. Clinical Cancer Research, 1127 (2019). DOI: 10.1158/10780432.CCR-19-1127

[7] Erickson B, Akkus Z, Sedlar J, Korfiatis P. Data From LGG-1p19q Deletion. The Cancer Imaging Archive. (2017) DOI: 10.7937/K9/TCIA.2017.dwehtz9v

[8] Fedorov A, Beichel R, Kalpathy-Cramer J, Finet J, Fillion-Robin J C, Pujol S, et al. 3D slicer as an image computing platform for the quantitative imaging network. Magn Reson Imaging (2012);30: 1323-41.

[9] Griethuysen J J M, Fedorov A, Parmar C, Hosny A, Aucoin N, Narayan V, Beets-Tan R G H, Fillon-Robin J C, Pieper S, Aerts H J W L. 
Computational Radiomics System to Decode the Radiographic Phenotype. Cancer Research, (2017), 77(21), e104-e107. https://doi.org/10.1158/0008-5472.CAN-17-0339

[10] Yang J, Ward M O, Rundensteiner E A, Huang S. Visual Hierarchical Dimension Reduction for Exploration of High Dimensional Datasets. Joint EUROGRAPHICS - IEEE TCVG Symposium on Visualization, (2003)

[11] Yin H. Nonlinear Dimensionality Reduction and Data Visualization: A Review. International Journal of Automation and Computing, (2007), N. 4 vol. 3, 294-303

[12] Shorten C. and Khoshgoftaar T M. A survey on image data augmentation for deep learning. (2019) Journal of Big Data, 6(1):60.

[13] Moreno-Barea, F J, Strazzera F, Jerez J M, Urda D and Franco L. Forward noise adjustment scheme for data augmentation. In 2018 IEEE Symposium Series on Computational Intelligence (SSCI), (2018), pages 728-734. IEEE.

[14] G. Bonaccorso, Machine Learning Algorithms: A Reference Guide to Popular Algorithms for Data Science and Machine Learning, Packt Publishing, Birmingham, UK, (2017).

[15] Berrar D. Cross-validation. Encyclopedia of Bioinformatics and Computational Biology, pp. 542-545. Academic Press, Oxford 2019. https://doi.org/10.1016/B978-0-12-809633-8.20349-X

[16] Xing L, Li R, Napel S, Rubin D. Radiomics and Radiogenomics: Technical Basis and Clinical Applications. Boca Raton, FL: CRC Press, 2019

[17] Lu C-F, Hsu F-T, Hsieh KL-C, Kao Y-CJ, Cheng S-J, Hsu JB-K, et al. Machine learning-based radiomics for molecular subtyping of gliomas. Clin Cancer Res. (2018). 24:4429-36. doi: 10.1158/1078-0432.CCR-173445

[18] Han Y, Xie Z, Zang Y, Zhang S, Gu D, Zhou M, et al. Non-invasive genotype prediction of chromosome $1 \mathrm{p} / 19 \mathrm{q}$ co-deletion by development and validation of an MRI-based radiomics signature in lower-grade gliomas. J Neurooncol. (2018) 140:297-306. doi: 10.1007/s11060-0182953-y

[19] Z. Kong, C. Jiang, Y. Zhang, S. Liu, D. Liu, Z. Liu, W. Chen, P. Liu, T. Yang, Y. Lyu, D. Zhao, H. You, Y. Wang, W. Ma, F. Feng. Thin-slice magnetic resonance imaging-based radiomics signature predicts chromosomal 1p/19q Co-deletion status in grade II and III gliomas. Front. Neurol., 11 (2020), p. 551771

[20] van der Voort, S. R. et al. Predicting the $1 \mathrm{p} / 19 \mathrm{q}$ co-deletion status of presumed low grade glioma with an externally validated machine learning algorithm. Clinical Cancer Research, 1127 (2019)

[21] Shboul ZA, Chen J, M Iftekharuddin K. Prediction of molecular mutations in diffuse low-grade gliomas using MR imaging features. Sci Rep.(2020);10(1):3711.

[22] M. Decuyper, S. Bonte, K. Deblaere, and R. Van Holen. Automated mri based pipeline for glioma segmentation and prediction of grade, idh mutation and 1p19q co-deletion,arXiv preprint arXiv:2005.11965, (2020).

[23] Fukuma R, Yanagisawa T, Kinoshita M, et al. Prediction of IDH and TERT promoter mutations in low-grade glioma from magnetic resonance images using a convolutional neural network. Sci Rep (2019);9:20311 CrossRef Medline

[22] Kocak B, Durmaz ES, Ates E, et al. Radiogenomics of lower-grade gliomas: machine learning-based MRI texture analysis for predicting 1p/19q codeletion status. Eur Radiol (2020);30:877-86 CrossRef Medlin 